\title{
Schema Design of Communication Module in Highway Monitoring System: A Case Study
}

\author{
http://dx.doi.org/10.3991/ijoe.v10i1.3050 \\ Ning Chen, Xiao-ping Song, Yi Liu \\ College of Computer Science, Xi' an Polytechnic University, Xi' an, China
}

\begin{abstract}
Because of an abundance of nodes in highway monitoring system and high complexity of Highway Monitoring System, a UDP-based communication module in Highway Monitoring System was designed, which can ensure consistency of information between nodes by means of the control command standardization and real-time interaction based on UDP protocol. Finally, the communication module was realized, and experiments showed that the design is feasible and has some flexibility.
\end{abstract}

Index Terms - Highway Monitoring System; Communication Module; UDP protocol

\section{INTRODUCTION}

Nowadays, Highway plays an important role in the national economy. However, the urgent requirements for its normal operation and its creative potential have resulted in increasingly dependent on the highway monitoring system. The highway monitoring system has become an important field of scientific research which gains more concern, and more researchers make great efforts to develop and disseminate it, therefore, it is a secular trend of the 21 st-century intelligent transportation system [1].

The highway monitoring system can save people's time and money, reduce the number of traffic casualties, and improve the quality of people's lives with regard to the perspective of safety and service function [2]. So, it is claimed that highway monitoring system throughout the country is the basis for the modernization of future transportation industry, which will create greater social and economic benefits than the existing interstate highway system in the United States in the next 20 years, just as cement, asphalt and steel rail do. Therefore, the highway monitoring system is the irresistible trend of the $21 \mathrm{st}-$ century transportation system, an important field of scientific research worthy to be developed and disseminated [3].

\section{Overview of Highway Monitoring System}

The highway is enclosed, fully interchanged roadway, with the fast vehicles and heavy traffic. The perfect characteristics are "safe, fast, efficient, comfortable, and convenient". However, with the growing demand of traffic, congestion, accidents and pollution occurred, making the highway monitoring system to become the focus of attention of administrative authority in charge of the construction of transportation facilities [4].

The so-called monitoring, refers to real-time observation of traffic conditions [9], road surface, weather conditions and equipment conditions and other parameters which are sent to the monitoring center via the communi- cation system to adjust and control traffic, by means of data acquisition on the pavement and the roadside, testing equipment and artificial observation [5].

Overseas study on the Highway monitoring system commenced early, and the nowadays Highway monitoring system also have been perfect, for example, United States, Canada, Europe and Japan lead in the Highway monitoring system [6]. United States commenced large-scale construction of highway since the 1950s, and endeavored in the operation and management of the highway after 1960, therefore, some of the city's highways installed various Highways monitoring system [8]. Currently, the computer was introduced into the Highway monitoring system, so as to achieve the optimum operation of the highway [7].

At present, there is a wide gap between highway monitoring system in China and foreign countries, with vehicle detectors, cameras, intelligence panels of low density in China. There are differences between the situation in China and the developed countries, for example, the poor performance of vehicles, complex traffic composition, so we should study new highway monitoring system suitable for China's conditions, and we not only learn the advanced experience of foreign countries, but also integrate with China's conditions.

\section{Highway Monitoring System}

Highway monitoring system is a application of advanced information technology, data transmission technology, electronic control technology and computer processing technology in management system for Highway transportation, which can give full scope to real-time, accurate and efficient management system for Highway transportation. Application of highway monitoring system in a comprehensive monitoring and controlling highway will play an extremely important role in the normal operation on the highway and efficiency of the highway.

Highway monitoring system is also a computer system with C / S (Client / Server) mode. The server is responsible for computing and information storage, and the client is responsible for handling the user interface. Server and Client can be connected to a LAN in monitoring center by means of the computer network equipment. In addition to connecting server and client in a LAN in monitoring center, monitoring center controls large-screen projectors, video switching matrix, video walls, map board by way of the associated computer device. 


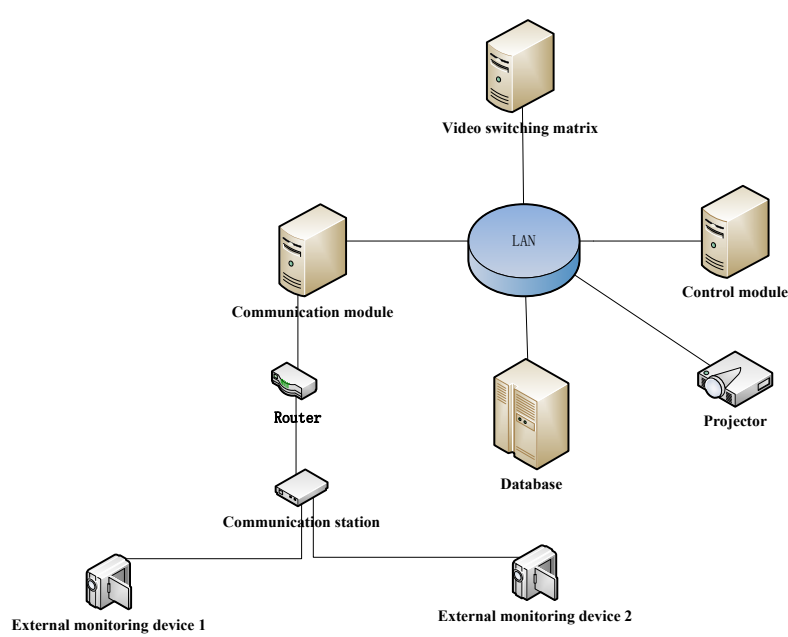

Figure 1. Highway monitoring system

\section{UDP-BASED COMMUNICATION}

From a point of view of information flow in Highway monitoring system, the communication module is the entry of the external information into the system, and the exit of remote control command to external device, which plays the important role of Information transmission in Highway monitoring system. Its main functions are responsible for sending control and feedback Information to external monitoring device, receiving control and feedback Information from external monitoring device and system clock proofreading etc...

The communication module provides communication channel for data Interchange between external monitoring device and monitoring center. Information collected by external monitoring device is sent to the nearest communication station through the dedicated line modem, and then information will upload to the monitoring center through Computer Network.

Communication Protocol: In the communication module, we should determine the communication protocol, a port for each external device, the communication parameters of each port, such as: communication rate, data bits, and parity bits, stop bits, etc.

- Communication parameters: transmission rate from $1200 \mathrm{bps}$ to $19.6 \mathrm{Kbps}$, 8 bit data bit, no parity bit, 1 bit start bit, 1 bit stop bit, no hardware and software flow control.

- The packets definition: STX-0x02 (header of frame), 0x03 (end of frame), ESC-0x1B (escape character). While Sending a frame, if a character between the header and the end of the frame byte is equal to the STX, ETX or ESC, its meaning ought to change, for example, STX will turn into ESC and 0xE7; ETX will turn the ESC and 0xe8; ESC will turn into ESC and $0 \mathrm{x} 00$.

- Packet Format

\begin{tabular}{|l|l|l|l|l|}
\hline STX & ADDR & INFO & CRC & EXT \\
\hline
\end{tabular}

INFO part is the variable length information field. The contents of the command and response are in this area.

ADDR is address of external device, with the range of 01 to 99,00 as the broadcast address.
CRC generator polynomial is $\mathrm{G}(\mathrm{X})=\mathrm{X}^{\wedge} 16+\mathrm{X}^{\wedge} 12$ $+\mathrm{X}^{\wedge} 5+1$, while CRC is computed excluding the STX.

Other packet formats are given below:

- To take device's failure information

Send:

\begin{tabular}{|c|c|c|c|c|}
\hline STX & ADDR & '01' & CRC & EXT \\
\hline \multicolumn{5}{|c|}{ Response: } \\
\hline STX & ADDR & STATE & CRC & EXT \\
\hline
\end{tabular}

- Display device list

Send:

\begin{tabular}{|c|c|c|c|c|}
\hline STX & ADDR & '98' & $\mathrm{CRC}$ & EXT \\
\hline \multicolumn{5}{|c|}{ Response: } \\
\hline STX & ADDR & OK & $\mathrm{CRC}$ & EXT \\
\hline
\end{tabular}

- Change the display mode of device

Send:

\begin{tabular}{|c|c|c|c|c|}
\hline STX & ADDR & “04' & CRC & EXT \\
\hline
\end{tabular}
\begin{tabular}{|c|c|c|c|c|}
\hline Response: \\
STX & ADDR & OK & CRC & EXT \\
\hline
\end{tabular}

- Adjustment of display intensity of device.

Send:

\begin{tabular}{|l|l|l|l|l|l|l|l|}
\hline STX & ADDR & '05' & RED & GREEN & BLUE & CRC & EXT \\
\hline
\end{tabular}

Response:

\begin{tabular}{|c|c|c|c|c|}
\hline STX & ADDR & OK & CRC & EXT \\
\hline
\end{tabular}

- To take the display mode and current display intensity of device

Send:

\begin{tabular}{|c|c|c|c|c|c|}
\hline STX & ADDR & '06' & CRC & & $\mathrm{XT}$ \\
\hline \multicolumn{6}{|c|}{ Response: } \\
\hline STX & ADDR & BMODE & BRI & CRC & EXT \\
\hline
\end{tabular}

UDP-based Communication: UDP-based communication is used in the highway monitoring system. When Computer in communication station has received information collected by the external monitoring device, the information is passed to the monitoring computer in monitoring center by means of UDP packets, then the monitoring computer writes the information into the server, finally the information is displayed on the map board by graphics workstation. In addition, video surveillance workstation and toll stations use UDP-based communication. Therefore, a brief introduction on "connectionless-oriented" protocol UDP is given below [10].

"connectionless-oriented" means that data packets are directly sent regardless of state on the other side, without a preestablished connection with each other before the formal communication [16]. This is very similar to text messages in mobile phone: when you send text messages, you only need to enter the recipient's mobile phone number, regardless of the recipient's service state [11].

UDP (User Datagram Protocol) is a protocol corresponding to TCP [18]. It is a connectionless-oriented protocol, which can directly send data packet, without preestablishing a connection with each other $[14,20]$. 
UDP is only suitable for application with sending small amounts of data and the fewer requirements for reliability [19]. For example, we often use the "ping" command to test TCP / IP communication between two computers. Principle of "ping" command is that UDP packet is sent to the computer on the other side, and then it confirmed receipt of UDP packets, in other words, if a message which denotes that the data package has arrived, is feedback in a timely manner, the network is Interconnection. For example, with default setting, a "ping" operation sends four packets. It is well known that four packets are sent, four packets should be received. (Because the other computer will send back confirmation packets as long as it receives a packets). This fully shows that the UDP protocol is a connectionless-oriented protocol, without a process of establishing a connection. Because of no a process of establishing a connection, the UDP protocol is efficient; in the same way, because of this, its reliability is lower than the TCP protocol [15]. For example, QQ, a chat application, employ UDP-based message, so sometimes the message maybe is lost [13].

UDP-based communication process is shown in Fig.2 [12]. On the server side, the server uses AF-INET protocol family to create a UDP datagram socket, with the socket type SOCK-DGRAM. The API function below will create a UDP datagram sockets:

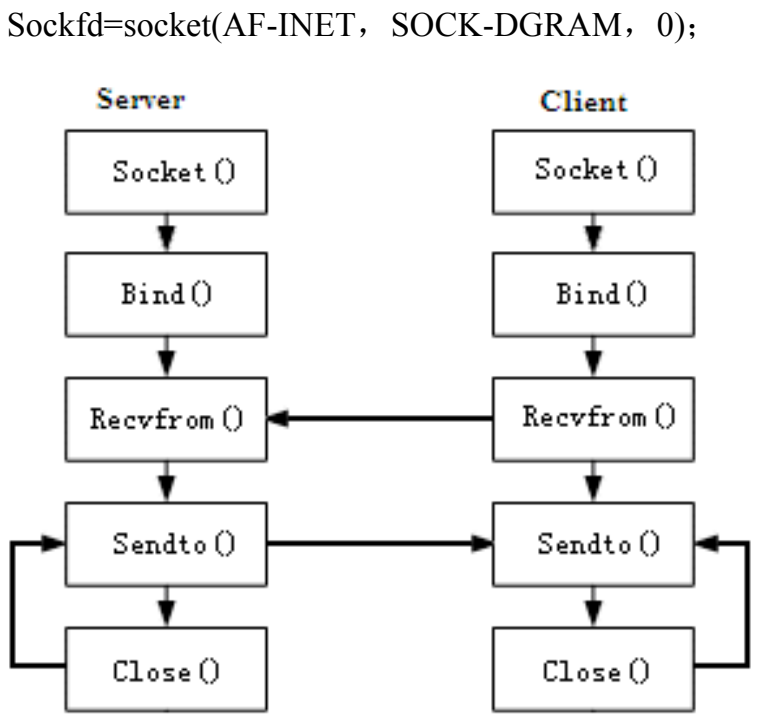

Figure 2. UDP-based communication process

The server then calls the bind function to bind a port to the UDP datagram socket. Due to have no use for establishing a connection, the server can be waiting for the UDP datagram which was sent by the client by means of calling recvfrom function on the specified port. The client firstly creates a UDP datagram socket by the socket function, with a port number assigned by the operating system. Since then, the client can use the sendto function to send a UDP packet to a specified address. Once server received the UDP packet, the recvfrom function will return. After server has processed the UDP packet, then server will return the results to the client by the sendto function.

Sendto and recvfrom function are defined as follows:

- The sendto function can send data in application buffer to the specified address space of the receiving process. Its prototype is: \#include $<$ sys/socket $\mathrm{h}>$

int sendto(int sockfd, const void *msg, int len, unsigned int flags, const struct sockaddr*to , int tolen);

- The recvfrom function can receive data in socket buffer and send it to the user process in the following form:

\#include $<$ sys/socket $\mathrm{h}>$

int recvfrom(int sockfd, const void *buf, int len, unsigned int flags, const struct sockaddr *from, sockln_t*addrlen);

\section{Communication Module}

The TNMUDP Control is introduced from NetMasters by Delphi. The programmer does not need to grasp the details of the UDP protocol, UDP-based communication can be implemented [17].

The TNMUDP Control's attributes:

- Loca1Port: This attribute is used to specify the local port. This property cannot be modified during operation.

- RemoteHost: This attribute is used to specify the computer to which the UDP packet is sent, a host name or IP address.

- RemotePort: This attribute is used to specify the port number used on the other computer.

The TNMUDP Control's primary methods:

- ReadBuffer(var Buff: array of char; var Length:integer);

The method is for receiving data and the received data is placed in a buffer. The Length parameter specifies the length of the buffer. If the Length of the buffer is too small, it will trigger the "OnBuffe Invalid" event.

- ReadStream(DataStream:TStream);

The method is for receiving data and the received data is placed in a stream specified by DataStream parameters. If the stream specified by DataStream parameter does not exist, it will trigger OnStreamInvalid event.

- $\quad$ sendStream(DataStream:TStream);

The method sends data specified by DataStream parameter.

Server is Windows 2000 Advance Server, and client is Windows XP Professional, with multitasking capabilities, multi-level system fault tolerance. Fig. 3 shows interface of communication module.

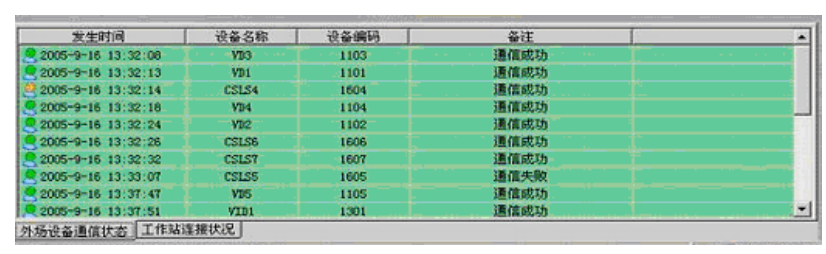

Figure 3. Communication Module

\section{CONCLUSION}

Highway monitoring system is a complex system and a systematic project, which involved the computer network technology, computer control technology, distributed database technology, component technology, communica- 
tions technology, graphics, image processing technology and many other aspects. The paper begins with practical requirements of highway monitoring system, followed by analysis of status in quo, requirement and characteristics of the highway monitoring system. Then the UDP-based communication in highway monitoring system is introduced, including: development platform, the software development and command packet format etc. The work not only is helpful for the future development of similar highway monitoring system, but also there are a certain significance for the development of monitoring systems for application in other areas.

\section{ACKNOWLEDGMENT}

This work is supported by Foundation of the Education Department of ShaanXi Province (2012JK0931), Guidance Project of China National Textile Industry Council (2010076), Xi'an Polytechnic University Foundation Research Project (XGJ07008), Student Innovative and Venture Training Program (201209114) and Foundation of Xi'an Polytechnic University PHD Scientific Research (Grant No.BS0725).

\section{REFERENCES}

[1] Pletzer, F.; Tusch, R.; Boszormenyi, L.; Rinner, B. "Robust Traffic State Estimation on Smart Cameras", 2012 IEEE Ninth International Conference on Advanced Video and Signal-Based Surveillance (AVSS), page(s): 434 - 439.

[2] E.F. Berliner, "Acoustic Highway Monitoring", US Patent Nr. 6,195,608, February 2001

[3] Highway Monitoring (HI-MONI), http://hi-moni.ftw.at. 2013.

[4] Stankova, K.; De Schutter, B. "On freeway traffic density estimation for a jump Markov linear model based on Daganzo's cell transmission model", 2010 13th International IEEE Conference on Intelligent Transportation Systems (ITSC), On page(s): $13-18$

[5] Mihai Caadariu, Monica Sabina Crainic, Mircea Poianaa, "Electronic Monitoring and Information System for Road Traffic" Roads Bridges Magazine no. 72 (141), pp.6-14, June 2009.

[6] Mihai Caadariu, Mircea Poiană, Monica Sabina Crainic, "SMITR Road Traffic Monitoring and for Highways and National Roads Part I" Roads Bridges Magazine no. 79 (148), pp. 18-22, January 2010.

[7] Mihai Caadariu, Mircea Poianaa, Monica Sabina Crainic, "SMITR Road Traffic Monitoring and for Highways and National Roads Part II" Roads Bridges Magazine no. 80 (149), pp. 6-9, February 2010.

[8] Tamersoy, B.; Aggarwal, J.K. "Robust Vehicle Detection for Tracking in Highway Surveillance Videos Using Unsupervised Learning", , 2009. Sixth IEEE International Conference on Advanced Video and Signal Based Surveillance( AVSS '09),page: 529 - 534 http://dx.doi.org/10.1109/AVSS.2009.57

[9] N. J. Ferrier, S. M. Rowe, and A. Blake. Real-time traffic monitoring. Proceedings of the Second IEEE Workshop on Applications of Computer Vision, page: 81-88, 1994.

[10] Jusak, J.; Harris, R.J. "Study of UDP-based Internet traffic: Longrange dependence characteristics", Australasian Telecommunication Networks and Applications Conference (ATNAC), 2011, page: $1-7$

[11] Min Zhang; Dusi, M.; John, W.; Changiia Chen, "Analysis of UDP Traffic Usage on Internet Backbone Links,". Ninth Annual International Symposium on Applications and the Internet (SAINT '09). pp.280-281, July 2009
[12] J. Postel, "User Datagram Protocol", RFC768, 1980

[13] Wang Jigang, Gu Guochang, Xu Lifeng and Wang Chen, Research and Design of Reliable Data Transfer Based on UDP. Computer Engineering and Applications. No.15, 2006. pp:113116.

[14] Zhou Peng, Wang Ya, A Research Based on the UDP and Reliable Real-time Transmission of Data, Computer and Network. No.20, 2008. pp: 69-71.

[15] Wang Haijun, Liu Caixia; Cheng Dongnian, Analysis and Research of a Reliable Transmission Protocol Based on UDP. Application Research of Computers. No.11, 2005. pp: 181-183.

[16] Lu Hongwei, How to Process the Packet Lost and Out of Order Based on UDP Transport Protocol. Computer Engineering and Applications. No.2, 2001. pp:48-50.

[17] LIU Z, HUANG J, LU X, et al. Data Dowload Based on Delphi Multi-thread Technology. Journal of Hohai University Changzhou, 2007, 3: 012.

[18] Yongmao Ren, Haina Tang, Jun Li and Hualin Qian, 2009. Performance Comparison of UDP-based Protocols Over Fast Long Distance Network. Information Technology Journal, 8: 600-604. http://dx.doi.org/10.3923/itj.2009.600.604

[19] Weihang Wang, Mingjie Tang, Yongmao Ren and Jun Li, 2010. Characterization and Evaluation of End-System Performance Aware Transport Schemes for Fast Long-Distance Optical Networks. Information Technology Journal, 9: 766-773. http://dx.doi.org/10.3923/itj.2010.766.773

[20] Z.M. Mekkakia , S. Selka and A. Benyettou , 2004. Behavior of TCP and UDP Flows with Differentiated Services . Journal of Applied Sciences, 4: 24-27. http://dx.doi.org/10.3923/jas.2004.24.27

\section{AUTHORS}

Ning Chen is associated professor of computer software and theory, Xi'an polytechnic university in 710048 Xi'an. He received BS (1992) and MS (1996) degrees in microelectronics from Xi'an JiaoTong University, China. $\mathrm{He}$ received the $\mathrm{PhD}$ degree in computer software and theory from Xi'an JiaoTong University in 2006. Since 2007, he has been a faculty member of Xi' an polytechnic university. His research interests include Software Process Modeling, Process and Workflow Management, Patterns and Frameworks, Requirements Engineering, Automated Software Specification, Component-Based Software Engineering, Automated Software Design and Synthesis. He received top prize for progress in electronic industry (970203) from ShaanXi Electronic Bureau in 1997. (email: chennvictor@gmail.com).

Xiao-ping Song is now an undergraduate in Xi'an polytechnic university , $710048 \mathrm{Xi}$ 'an, China (e-mail: 1963680316@qq.com).

Yi Liu, was with Xi' an JiaoTong University China. She is now with Xi'an polytechnic university, $710048 \mathrm{Xi}$ 'an, China (e-mail: chenn_victor@xpu.edu.cn).

This work is supported by Foundation of the Education Department of ShaanXi Province (2012JK0931), Guidance Project of China National Textile Industry Council (2010076), Xi'an Polytechnic University Foundation Research Project (XGJ07008), Student Innovative and Venture Training Program (201209114) and Foundation of Xi'an Polytechnic University PHD Scientific Research (Grant No.BS0725). Submitted, July, 8, 2013. Published as resubmitted by the authors on August, 16, 2013. 